\title{
Outcome of free gracilis muscle transfer for the restoration of elbow flexion in traumatic brachial plexus palsy
}

 \\ Anar Keremov, MD ${ }^{1}$, Sinan Adıyaman, MD $^{1}{ }^{10}$, Sırrı Sinan Bilgin, MD $^{1}$ \\ 'Department of Orthopedics and Traumatology, Ankara University, Faculty of Medicine, Ankara, Turkey \\ ${ }^{2}$ Department of Hand Surgery, Kanuni Training and Research Hospital, Trabzon, Turkey \\ ${ }^{3}$ Department of Orthopedics and Traumatology, Hand and Microsurgery \& Orthopedics and Traumatology Hospital, Izmir, Turkey \\ ${ }^{4}$ Department of Hand Surgery, Ankara City Hospital, Ankara, Turkey
}

Traumatic brachial plexus palsy (TBPP) is a devastating injury that significantly affects the upper extremity functions and results in serious disability. Surgical reconstruction is closely related to the severity of the injury and the time elapsed after the trauma. Microneural and/or free functional muscle transfer reconstruction can be performed in patients admitted in the early period. ${ }^{[1-3]}$ Tendon transfers, pedicle or free muscle transfers are the treatment options in patients with delayed TBPP and reserved for cases where nerve repair or reconstruction fails. ${ }^{[1,4]}$ Doi et al. ${ }^{[5]}$ reported successful results using free muscle transfer to restore limb functions in TBPP patients in 1991. The same team defined double free muscle transfer in 1995. ${ }^{[6]}$

Received: May 01, 2021

Accepted: June 11, 2021

Published online: November 19, 2021

Correspondence: Seyyid Şerif Ünsal, MD. Kanuni Eğitim ve Araştırma Hastanesi, El Cerrahisi Kliniği, 61290 Yomra, Trabzon, Türkiye.

E-mail: seyyid.unsal@hotmail.com

Doi: $10.52312 /$ jdrs.2021.225

Citation: Armangil M, Ünsal SS, Yıldırım T, Bezirgan U, Keremov A, Adıyaman S, et al. Outcome of free gracilis muscle transfer for the restoration of elbow flexion in traumatic brachial plexus palsy. Jt Dis Relat Surg 2021;32(3):633-641.

(อ2021 All right reserved by the Turkish Joint Diseases Foundation

This is an open access article under the terms of the Creative Commons Attribution-NonCommercial License, which permits use, distribution and reproduction in any medium, provided the original work is properly cited and is not used for commercial purposes (http://creativecommons.org/licenses/by-nc/4.0/).

\section{ABSTRACT}

Objectives: The aim of this study was to evaluate the functional outcomes of patients with free gracilis muscle transfer (FGMT) for the restoration of elbow flexion.

Patients and methods: Between January 2012 and January 2019, a total of 16 patients (13 males, 3 females; mean age: $27.3 \pm 11.7$ years; range: 18 to 53 years) who underwent FGMF to achieve elbow flexion after traumatic brachial plexus palsy (TBPP) were retrospectively analyzed. Data including demographic and clinical characteristics of the patients, etiology, affected side, injury level, accompanying injuries, time between injury and surgery, follow-up time, complications, whether nerve reconstruction and artery repair were performed previously, and details of the procedure were recorded. The outcome measures were elbow range of motion in degrees, muscle strength, and Disabilities of the Arm, Shoulder and Hand (DASH) and Short Form-36 (SF-36) scores.

Results: The mean follow-up was $30 \pm 11.5$ (range, 24 to 42 ) months. Two patients had C5-C8 injuries, two patients had C6-T1 injuries, and all other patients had injuries to the C5-T1 roots. Muscle strength was M3/M4 in 11 (68.8\%) patients, M2 in two (12.5\%) patients, and $\mathrm{M} 0$ in one $(6.2 \%)$ patient. The median active range of motion of the elbow joint in patients with successful results (M3 and above) was measured as 75 (range, 30 to 100) degrees. A statistically significant improvement was observed in the pre- and postoperative DASH scores and in some SF-36 subscale scores of patients with successful results.

Conclusion: Free gracilis muscle flap is a reliable option in the restoration of elbow flexion in patients with TBPP. Although there is an improvement in functional results, disability, and quality of life, there may be no change in patients' mental status and pain.

Keywords: Brachial plexus paralysis, elbow flexion, free muscle transfer, neurotization.

Elbow flexion is the most important function that needs to be restored in TBPP patients. ${ }^{[7]}$ For free functional muscle transfer, gracilis, rectus femoris, and latissimus dorsi muscles are preferred as the muscle donors. The latissimus dorsi and 
rectus femoris muscles do not have sufficient excursion and pedicle length such as the gracilis muscle. The latissimus dorsi lacks the distal tendinous portion for good repair of the biceps tendon stump. ${ }^{[6-8]}$ Currently, free gracilis muscle flap (FGMF) is frequently used for restoration of elbow function owing to its thin muscle body, sufficient strength and excursion, sufficient length, and easily obtainable neurovascular pedicle. ${ }^{[9]}$

In the present study, we aimed to evaluate the functional outcomes of patients with free gracilis muscle transfer for the restoration of elbow flexion.

\section{PATIENTS AND METHODS}

This single-center, retrospective study was conducted at Ankara University Faculty of Medicine, Department of Hand Surgery between January 2012 and January 2019. Patients over 18 years of age who had a follow-up for at least 12 months postoperatively and had complete data were included in the study. Patients who had loss of range of motion due to elbow trauma, who were under 18 years of age, who had a follow-up period of less than 12 months, and who underwent additional surgical procedures such as Steindler flexorplasty to increase elbow flexion were excluded from the study. Finally, a total of 16 patients (13 males, 3 females; mean age: $27.3 \pm 11.7$ years; range: 18 to 53 years) who underwent FGMF were included. A written informed consent was obtained from each patient. The study protocol was approved by the Ankara University Faculty of Medicine Ethics Committee (Date/no. 17.06.2020/I5-309-20). The study was conducted in accordance with the principles of the Declaration of Helsinki. The electronic patient documentation system was used to identify patient demographics, etiology, affected side, injury level, accompanying injuries, time between injury and surgery, follow-up time, complications, and whether nerve reconstruction and artery repair were performed previously. Furthermore, details of the procedure, including the origin and insertion of the FGMF, the preferred donor nerve, and need for a nerve graft, were also determined.

The outcome measures were elbow range of motion in degrees, muscle strength, and Disabilities of the Arm, Shoulder and Hand (DASH) and Short Form-36 (SF-36) scores. The active range of elbow motion was measured using a standard goniometer with the patient in the upright position. Postoperative muscle strength of the patients was evaluated according to the Modified British Medical Research Council (mMRC) questionnaire. Patients with at least M3 muscle strength were considered to have a successful result. The Turkish versions of the DASH and SF-36 questionnaires were used to evaluate the functional status and quality of life of the patients before and after surgery. The pre- and postoperative scores were calculated and recorded by orthopedic surgery residents.

\section{Surgical technique}

Surgery was performed under general anesthesia and in the supine position. Two surgical teams performed the operation simultaneously. While one team harvested the free gracilis flap, the other team prepared the donor site and explored the donor artery, vein, and nerve.

A line was drawn slightly inferior to the adductor longus going to the medial femoral condyle while the hip was in a slightly flexed and externally rotated position. A $6 \times 10 \mathrm{~cm}$ diameter elliptical skin island containing the adductor longus fascia for the more reliable skin pedicle was drawn along this line on the proximal third of the thigh. The pedicle was located between the cleavage of the gracilis muscle and the adductor longus muscle proximally. The pedicle should be dissected proximally as much as possible. To ensure the gracilis muscle was placed in the arm at
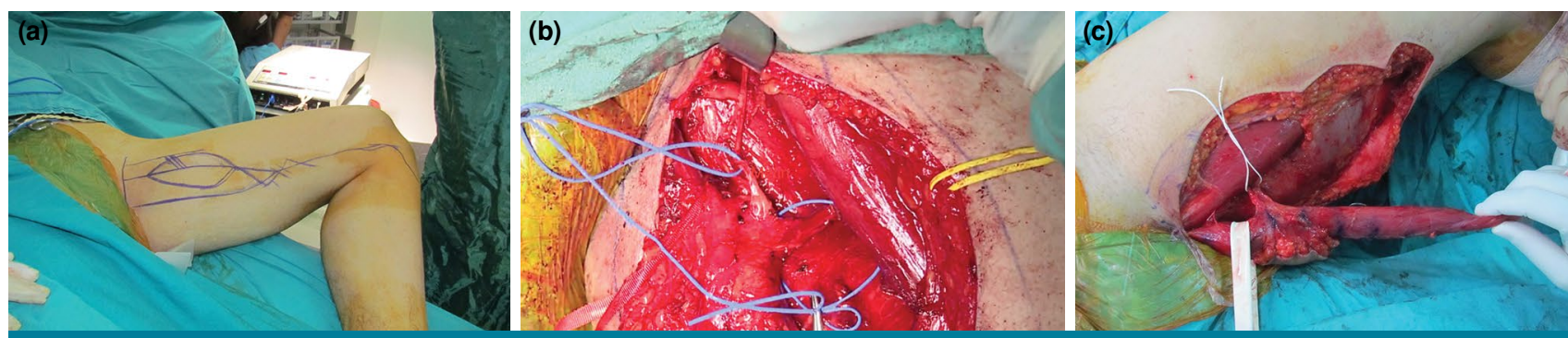

FIGURE 1. (a) Surgical design of the donor site before operation. (b) Neurovascular pedicle of gracilis muscle. (c) The gracilis was dissected from the thigh and tag sutures were placed $5 \mathrm{~cm}$ apart from each other. 
the proper tension, tag sutures were placed $5 \mathrm{~cm}$ apart from each other, when the muscle was in the longest position (Figure 1). The origin of the gracilis muscle was dissected proximally until its ischial attachment, including the tendinous part. A longitudinal incision was made over the attachment of the pes anserinus on the proximal anteromedial tibia to separate insertion. To keep the ischemia time short, the flap's vascular pedicle and nerve were divided, when the recipient side was ready for flap inset.

A deltopectoral incision was made at the recipient side, and the cephalic vein was found and protected. The pectoralis minor muscle was divided after placing a tag suture at the site of insertion to the coracoid process. The axillary artery and its branch thoracoacromial trunk were, then, explored. In our routine practice, we typically use the venae comitantes of the thoracoacromial artery as the donor vein, followed by the cephalic vein. We usually prefer the spinal accessory nerve (SAN) as the donor nerve. We prefer the medial pectoral nerve, the phrenic nerve, or intercostal nerves in patients whose SAN is not available.

The gracilis was attached to the acromion or lateral aspect of the clavicle proximally, allowing coaptation of the anastomosis of the vascular pedicle and the transfer of the nerve. The gracilis muscle was, then, placed subcutaneously in the anterior aspect of the arm and sutured to the biceps tendon distally.
The extensor digitorum communis tendons or flexor digitorum profundus tendons were preferred as insertion in patients in whom finger movement was desired in addition to elbow flexion (Figure 2). The appropriate tension of the muscle was adjusted with the help of tag sutures previously placed at $5 \mathrm{~cm}$ intervals. After adjusting the tension of the muscle, vascular anastomoses and nerve transfer were completed. Patients were immobilized in a long arm splint with the elbows in 1,000 of flexion to decrease the tension at the tendon attachment sites. Additional Velpeau $^{\mathrm{TM}}$ (Shenzhen Weprotex Technology Product Co., Ltd., Guangzhou, China) bandages were used to secure the arm.

\section{Postoperative period}

Flap circulation was monitored every hour for the first $24 \mathrm{~h}$ by checking capillary filling, color, and temperature of the skin island. Postoperatively, low-molecular-weight heparin and acetylsalicylate were administered. The flap examination was, then, performed every $2 \mathrm{~h}$ for the subsequent $48 \mathrm{~h}$. Meanwhile, hypothermia and hypovolemia were avoided and hemoglobin levels were kept above $10 \mathrm{mg} / \mathrm{dL}$. All patients were observed for at least a week.

A long arm splint and Velpeau ${ }^{\mathrm{TM}}$ bandage was used for six weeks. At the end of this period, the patients were referred to the physiotherapy department, and passive range of motion exercises were initiated.
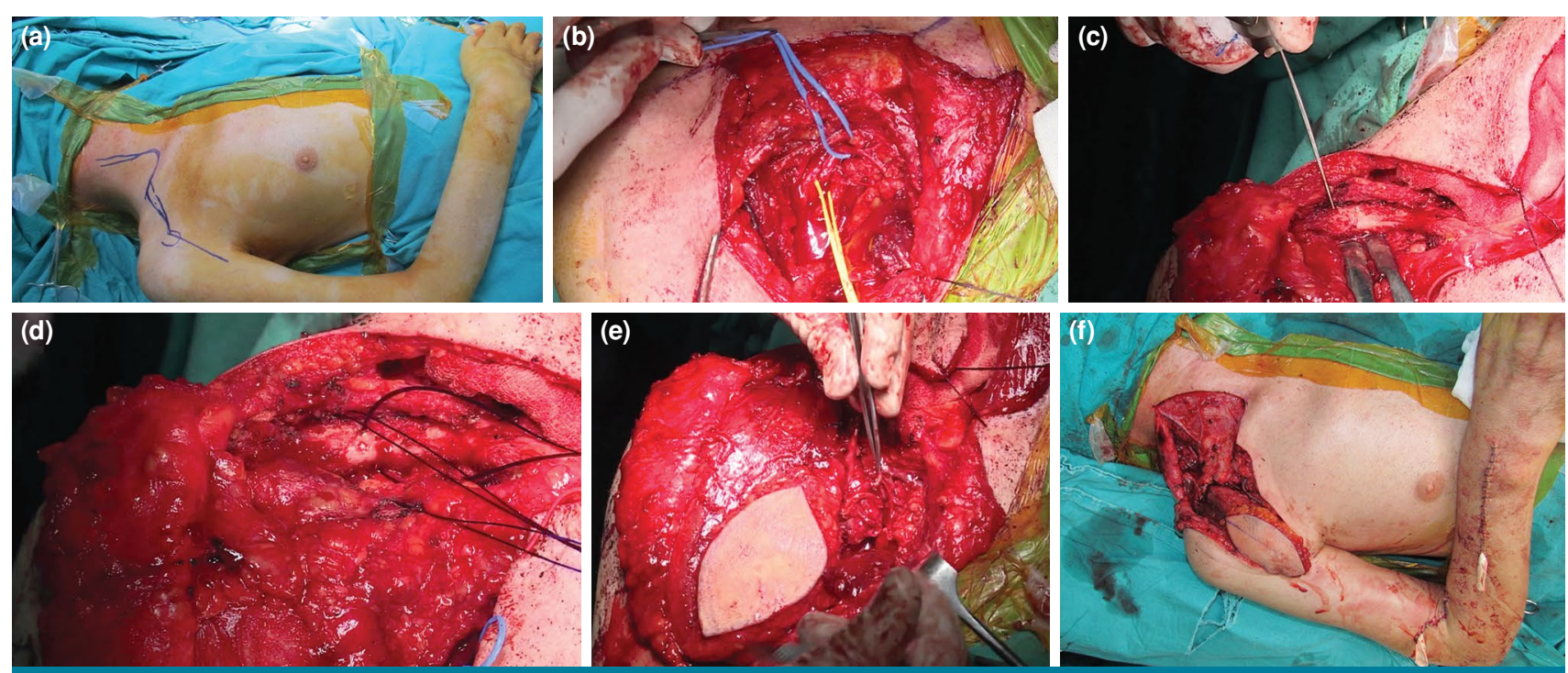

FIGURE 2. (a) Surgical design of the recipient site before operation. (b) The axillary artery and its branch thoracoacromial trunk were explored. (c, d) Preparation of lateral aspect of the clavicle proximally for the gracilis muscle was attached. (e, f) Gracilis muscle transferred to the arm. Proximal attachment was made onto the proximal clavicula. An end-to-end anastomosis of the vascular pedicle onto the thoracoacromial trunk was performed. The gracilis nerve was reinnervated with the spinal accessory nerve. 
The patients with SAN transfer were instructed to practice shoulder shrugs by pulling their shoulder backwards. A patient with intercostal nerve transfer was advised to perform deep breathing exercises. If contraction in the gracilis muscle was detected during the examinations or if reinnervation findings were detected in the electromyography (EMG), the patient was started with active range of motion exercises by eliminating gravity.

\section{Statistical analysis}

Statistical analysis was performed using the IBM SPSS version 23.0 software (IBM Corp., Armonk, NY, USA). Continuous data were expressed in
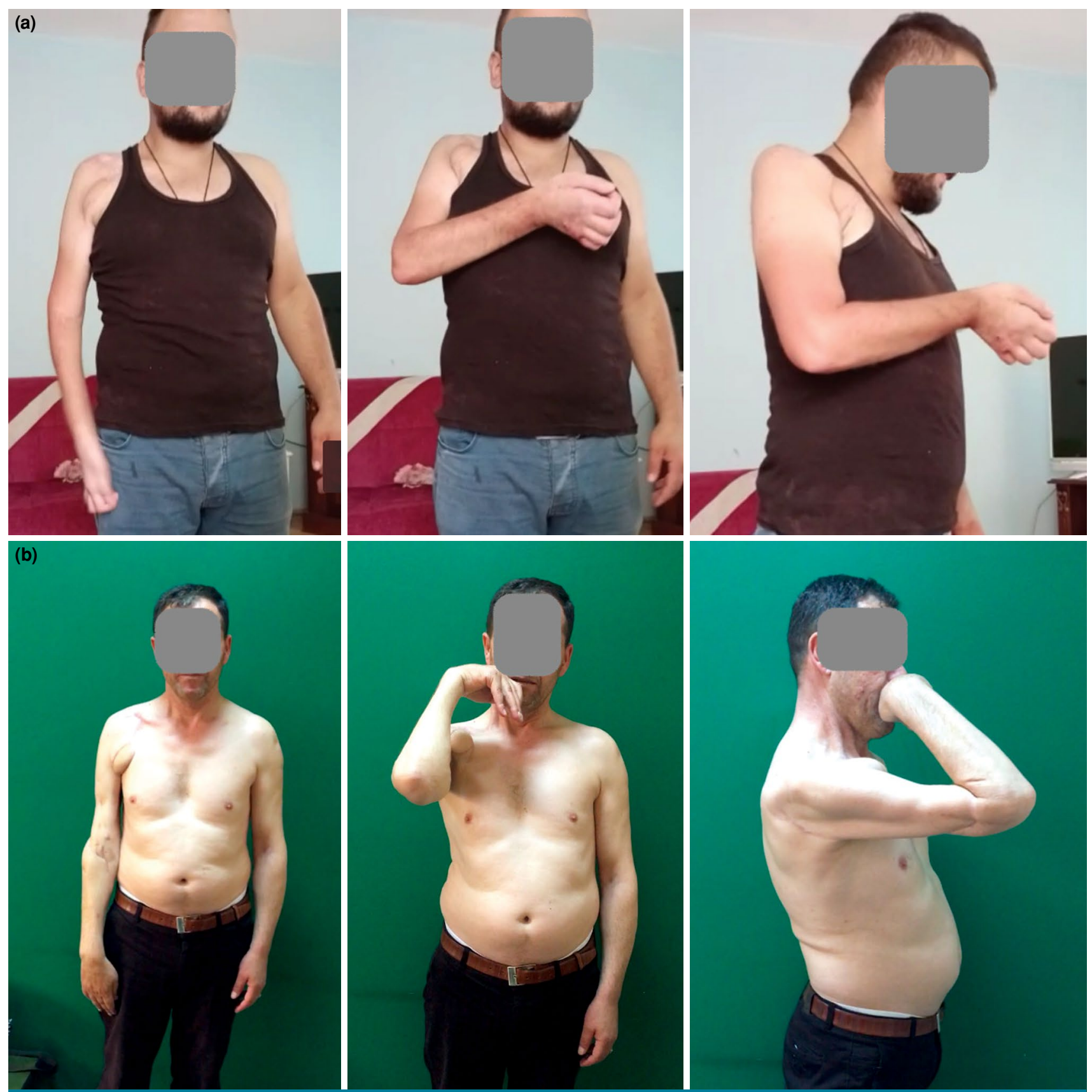

FIGURE 3. (a) A 20-year-old, motorcycle accident, TBPP. FGMF was reinnervated with spinal accessory nerve. He had M3+ elbow flexion at the final follow-up (at Month 16). (b) A 51-year-old, work accident, TBPP. He had M3+ elbow flexion at the final follow-up (Month 30).

TBPP: Traumatic brachial plexus palsy; FGMF: Free gracilis muscle flap. 
mean \pm standard deviation (SD), median (min-max), while categorical variables were expressed in number and frequency. The Shapiro-Wilk test was used to assess the data distribution. The Wilcoxon signed-rank test and the paired sample t-test were used to analyze and compare pre- and postoperative values of the patients. Differences between with and without arterial injury and repair and previous direct nerve surgery were compared either using the independent samples t-test or the Mann-Whitney test. A $p$ value of $<0.05$ was considered statistically significant.

\section{RESULTS}

The mean follow-up was $30 \pm 11.5$ (range, 24 to 42 ) months. The flap loss occurred in two (12.5\%) patients in the early postoperative period due to vascular problems. Two (12.5\%) patients had C5-C8 injuries, two $(12.5 \%)$ patients had C6-T1 injuries, and all other patients had injuries to the $\mathrm{C} 5-\mathrm{T} 1$ roots. The median delay from injury to free muscle transfer was 18 (range, 4 to 96) months. Seven patients had a motorcycle accident, five patients had motor vehicle accident, one $(6 \%)$ patient had a pedestrian accident, two $(12.5 \%)$ patients had a work accident, and one (6\%) patient had a bicycle accident. The SAN was used as the donor nerve in $12(75 \%)$ patients, the medial pectoral nerve in two $(12.5 \%)$ patients, the phrenic nerve in one $(6 \%)$ patient, and the intercostal nerves (fourth, fifth, and sixth nerves) in one (6\%) patient. The sensory branches of the intercostal nerve were simultaneously transferred to provide median nerve sensorial reconstruction. Nerve grafting was used in two $(12.5 \%)$ patients. The EMG is not used as a standard tool to evaluate gracilis muscle reinnervation in our clinical practice. On physical examination, contraction of the gracilis muscle was observed at an average of six months. Superficial postoperative infection was observed in only two (12.5\%) patients and these patients were treated with superficial debridement and intravenous antibiotics.

Muscle strength was M3/M4 in 11 (68.8\%) patients, M2 in two (12.5\%) patients, and M0 in one (6.2\%) patient (Figures 3 and 4). The median active range of motion of the elbow joint in patients with successful results (M3 and above) was measured as 75 (range, 30 to 100) degrees. A statistically significant improvement was observed in the preand postoperative DASH scores and in some of the SF-36 subscale scores of the patients with successful results. Clinical results of the patients are summarized in Table I.

Biceps tendon was used as insertion in eight (50\%) patients, forearm extensor tendons in four (25\%) patients, and forearm flexor tendons in four (25\%) patients. There was no statistically significant difference between the range of motion and muscle strength obtained between the two groups in which biceps and forearm muscles were used for insertion $(\mathrm{p}>0.05)$. In three patients, subclavian artery injury
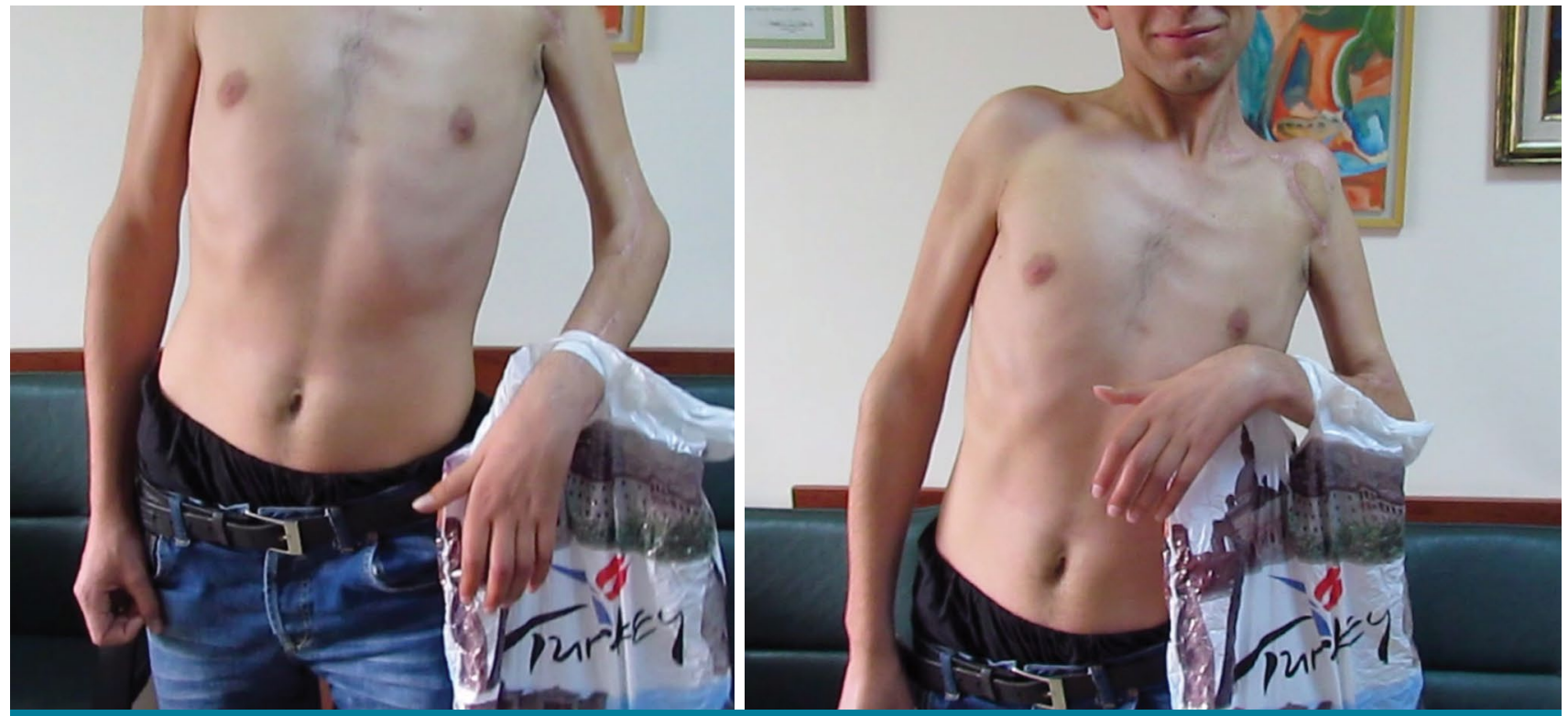

FIGURE 4. A 21-year-old, motorcycle accident, C5-C8 injury. FGMF was reinnervated with spinal accessory nerve. He could lift 1 -kg weight at the final follow-up (Month 32).

FGMF: Free gracilis muscle flap. 


\begin{tabular}{|c|c|c|c|}
\hline \multicolumn{4}{|c|}{$\begin{array}{l}\text { TABLE I } \\
\text { SF-36 scores before and after surgery }\end{array}$} \\
\hline \multirow[b]{2}{*}{ Outcome measures scores } & \multirow{2}{*}{$\begin{array}{c}\text { Preoperative } \\
\text { Mean } \pm S D\end{array}$} & \multirow{2}{*}{$\begin{array}{c}\text { Postoperative } \\
\text { Mean } \pm \text { SD }\end{array}$} & \multirow[b]{2}{*}{$p$} \\
\hline & & & \\
\hline $\mathrm{DASH}^{*}$ & $71.1 \pm 4.6$ & $48.5 \pm 14.0$ & 0.002 \\
\hline Elbow flexion (slope) ${ }^{*}$ & 0 & $62.9 \pm 41.4$ & 0.003 \\
\hline Elbow flexion strength $(\mathrm{kg})^{*}$ & 0 & $1.3 \pm 1.0$ & 0.003 \\
\hline \multicolumn{4}{|l|}{ SF-36 } \\
\hline Physical functioning ${ }^{*}$ & $56.1 \pm 2.9$ & $67.1 \pm 5.5$ & 0.001 \\
\hline Role physical ${ }^{*}$ & $0.0 \pm 0.0$ & $8.9 \pm 12.4$ & 0.025 \\
\hline Role emotional* & $7.1 \pm 14.1$ & $16.5 \pm 17.1$ & 0.066 \\
\hline Vitality* & $28.9 \pm 4.0$ & $41.8 \pm 8.5$ & 0.001 \\
\hline Emotional well-being ${ }^{\star *}$ & $33.1 \pm 4.0$ & $43.7 \pm 6.6$ & $<0.001$ \\
\hline Social functioning ${ }^{*}$ & $27.7 \pm 5.3$ & $44.3 \pm 7.9$ & 0.001 \\
\hline Pain* & $52.1 \pm 6.1$ & $53.3 \pm 6.7$ & 0.347 \\
\hline General health perception* & $51.8 \pm 4.6$ & $66.8 \pm 8.7$ & 0.001 \\
\hline $\begin{array}{l}\text { DASH: Disabilities of the Arm, Sh } \\
{ }^{*} \text { Wilcoxon signed-rank test; }{ }^{* *} \text { Paire }\end{array}$ & $\begin{array}{l}\text { land, SF-36: } \\
\text { st. }\end{array}$ & -36; SD: Star & \\
\hline
\end{tabular}

was detected. There was no statistically significant difference in the postoperative elbow flexion strength and range of motion of patients with and without arterial injury $(\mathrm{p}>0.05)$.

\section{DISCUSSION}

Traumatic brachial plexus palsy is usually seen in young active men and causes significant lifelong disability. The average age of occurrence has been reported to be between 21 and 34.3 years, ${ }^{[5,9]}$ and motorcycle accidents have been shown to be the most common cause in its etiology. ${ }^{[10]}$ Similar to the literature, the median age of patients was 23 years in our study, and the most common etiological cause was motorcycle accidents $(n=7,43.8 \%$, followed by other motor vehicle accidents $n=5,31.3 \%$ ). The patients who developed TBPP after a motorcycle accident were all males under the age of 30 years. Employment and pain management appear to be the primary concerns of these young individuals; however, there appears to be a disparity between patients' needs and what reconstruction surgery can achieve. In our study, although there was an improvement in the functional results, disability, and quality of life, there may be no change in the patients' mental status and pain. ${ }^{[1,12]}$ Therefore, a comprehensive preoperative consultation should be provided, considering the patients' expectations after reconstruction with FGSF.

Recovery of elbow function is the primary goal of treatment in patients with TBPP. As hand and wrist functions are preserved in patients with upper truncus injuries, providing elbow function in these patients would ensure that the hand will be able to reach to the mouth for feeding and personal hygiene. In total brachial plexus injuries, providing elbow function would be a support to the uninjured arm. ${ }^{[1]}$ Successful results can be obtained with nerve repair, grafting, and nerve transfer within the first nine months after injury. Free muscle transfer should be considered as a treatment option in case of root avulsion injury, unsuccessful nerve reconstruction surgeries, and complete brachial plexus injury accompanied by excessive time after injury. ${ }^{[2,3]}$

In the literature, rectus femoris, latissimus dorsi, and gracilis muscles were used as free muscle flaps to restore elbow function after TBPP. ${ }^{[10,13-15]}$ The gracilis muscle has a thinner diameter and less force; however, the advantages are less donor site morbidity compared to other muscles, better excursion, and having sufficient length pedicles that can be obtained more easily. ${ }^{[6]}$ Terzis and Kostopoulos ${ }^{[16]}$ reported that the gracilis muscle was not strong enough for elbow flexion, despite sufficient excursion, and that more power would be obtained from the latissimus dorsi and rectus femoris muscles. However, many studies have achieved elbow flexion with M3 + and M4 strength with FGMF at rates varying from 68 to $100 \% \cdot{ }^{[10,14,17]}$ It has been reported that the fixation to the biceps tendon would not be strong enough due to the lack of the tendinous part of the distal of the latissimus dorsi, and weakness in knee extension 
strength would occur if the rectus femoris is used. ${ }^{[18]}$ Obtaining $\mathrm{M} 3$ + muscle strength in $68.8 \%$ of our cases is compatible with the literature. The median muscle strength obtained in patients with successful results in this study was 1.5 (range, 0.5 to 2.5 ) $\mathrm{kg}$. Providing elbow flexion strength in patients with complete TBPP provides a stable and controlled limb that does not remain in swing while walking and prevents balance. ${ }^{[19]}$

Doi $^{[20]}$ described the use of extensor digitorum communis tendons as tendon insertions to achieve simultaneous elbow flexion and finger extension. Some authors preferred the biceps tendon, flexor digitorum profundus, extensor digitorum communis and extensor pollicis longus as distal insertions. ${ }^{[7,910,13]}$ Maldonado et al. ${ }^{[17]}$ reported that if the distal insertion of the tendon was made to the forearm flexors or $10 \mathrm{~cm}-15 \mathrm{~cm}$ distal to the elbow joint, 15 to $20 \%$ more power would be obtained and the active range of motion would be greater. Some studies have used the biceps tendon as an insertion and have achieved high M4 muscle strength. ${ }^{[10,17,21]}$ An aggressive, continuous rehabilitation process that lasts for at least 18 months is required to achieve the desired muscle strength. ${ }^{[20]}$ In our cases, M3+ muscle strength was obtained in six patients in whom gracilis muscle was inserted to the biceps tendon. Unfortunately, functional finger motion could not be achieved in cases using forearm muscle insertions, but active elbow flexion was achieved in $75 \%$ of these cases. We attribute the inability to achieve functional finger movement to our inadequate implementation of the rehabilitation program recommended by Doi et al.,[22] which includes early range of motion exercises in the early postoperative period.

In FGMF, the ulnar and median nerve fascicles, the contralateral C7 root, the SAN, the phrenic nerve, the intercostal nerves, and the medial pectoral nerve have been used as donor nerves. ${ }^{[10]}$ In a study including 38 patients, Chung et al. ${ }^{[14]}$ showed that all three patients in whom the musculocutaneous nerve was used as the donor nerve and $78 \%$ of the 31 patients in whom the intercostal nerve was used achieved M4 muscle strength, but all four patients in whom the SAN was used only obtained M2 muscle strength. Maldonado et al., ${ }^{[17]}$ Kay et al. ${ }^{[21]}$ Barrie et al., ${ }^{[23]}$ and Estrella and Montales ${ }^{[24]}$ reported successful results using both the intercostal nerve and the SAN. In their study, Dodakundi et al. ${ }^{[25]}$ used only the SAN; 24 of their 36 patients had M4 muscle strength and 11 had M3 muscle strength. In another study including 42 patients, Yang et al. ${ }^{[26]}$ used the SAN $(n=40)$ and phrenic nerves $(n=2)$ and obtained
M4 muscle strength in 24 patients and $\geq \mathrm{M} 3$ muscle strength in 15 patients. The SAN, which is a pure motor nerve, is easily accessible, and the trapezius muscle is not denervated when its most distal part is used as the donor nerve. ${ }^{[24]}$ If the intercostal nerve is used, a larger surgical dissection is required and the surgical time increases. The number of axons in the anterior branch of the obturator nerve innervating the gracilis muscle was calculated to be 938 in a cadaveric study. ${ }^{[27]}$ The average number of axons in the SAN is 1,329 , the average number of axons in the medial pectoral nerve is 1,078 , and the average number of motor axons in a single intercostal nerve is 375 . To obtain sufficient innervation, the ratio of the axon number of donor and recipient nerves should be greater than 0.7. ${ }^{[27,28]}$ Therefore, at least two intercostal nerves should be used as donors. In our practice, we usually prefer the SAN, as it allows end-to-end repair, the need for grafts is minimal, and there is no donor site morbidity. However, we prefer other donor nerves, if the SAN has been used in previous surgeries. In our series, we had to use nerve grafts in only two patients. The donor nerves used in these patients were the fourth, fifth, and sixth intercostal nerves, and the SAN and M3+ muscle strength was obtained in both patients. Although it is recommended not to use nerve grafts, successful results can be still obtained.

The anterior circumflex, profunda brachii, brachial, axillary, thoracoacromial, and thoracodorsal arteries have been preferred as donor arteries. ${ }^{[10,13,14,17,21,23-25]}$ Satbhai et al. ${ }^{[29]}$ found that the subclavian artery was injured and distal circulation was provided by collateral circulation in four $(8 \%)$ patients in preoperative computed tomography angiography (CTA) for whom they planned double free muscle transfer. They used the thoracoacromial artery and cephalic vein as recipient vessels in the first stage, and the thoracodorsal artery and vein as recipient vessels in the second stage. In our study, we preferred the thoracoacromial artery in all patients owing to its convenient diameter and location. We preferred the venae comitantes of the thoracoacromial artery as the primary vein and the cephalic vein as the secondary vein. We performed two vein anastomoses in all patients. In three patients, subclavian artery injury was detected in CTA preoperatively. In these patients, the thoracoacromial artery was used for anastomosis, and there was no problem in flap circulation. In a patient who previously underwent subclavian arterial reconstruction with the saphenous vein, the flap anastomosis was performed to the vein graft in 
an end-to-side fashion. There was no problem with flap circulation in this patient. We can speculate that previous vascular surgery does not affect flap survival, but it makes the operation technically difficult. Reverse FGMT described by Bertelli ${ }^{[30]}$ can be performed in these patients. In this surgical method, the gracilis tendon is fixed to the acromion and the muscle core is sutured to the biceps distal tendon. Vascular repair is preferably done in an end-to-end fashion to the radial artery and cephalic vein.

The DASH score is used to evaluate functional results for muscle transfers. In our study, the mean postoperative DASH score was reported as $48.5 \pm 14.0$ in patients for whom elbow flexion was reconstructed with FGMF. An improvement between 19.7 and 52 points in DASH scores has been achieved after surgery in previous studies. ${ }^{[11,26,27,31]}$ Coulet et al. ${ }^{[12]}$ reported the mean postoperative DASH score as 32 in patients with total TBPP and 42 in patients with partial injury. ${ }^{[11]}$ The fact that patients with partial injuries had higher scores despite having hand function was attributed to the inability of these patients to accept their current disability. The mean pre- and postoperative DASH scores were $71.1 \pm 4.6$ and $48.5 \pm 14.0$, respectively, indicating a statistically significant improvement in our patients.

Dodakundi et al. ${ }^{[25]}$ evaluated the health status of patients who received two-staged FGMF using the SF-36 questionnaire. They reported an improvement in the physical function and physical role of the patients, but a worsening of their physical pain and no improvement in mental health, emotional role, vitality and social functioning. In the current study, we observed no significant improvement in the emotional role and pain, but there was an improvement in the social functioning, physical functioning, general health perception, physical role, emotional well-being and vitality subscales. Although elbow flexion was achieved in the most of our patients, mental status may not have improved, as the upper extremity function could not be fully restored due to the severity of the injury. Therefore, physicians should provide a detailed preoperative education to encourage realistic expectations and patients should be informed that there may not be a significant improvement in the level of pain after the operation.

The major complications of FGMF include flap loss, skin necrosis, infection, arterial thrombosis, clavicle fracture, bowstringing of the gracilis muscle, knee instability, and temporary peroneal nerve injury. ${ }^{[15,21,24,31-33]}$ The rate of flap loss has been reported at rates varying between 0 and $16.7 \%$ in the literature. ${ }^{[10]}$ We observed early flap loss due to venous thrombosis in two (12.5\%) patients in our study. Donor site morbidity was not observed in any of the patients. Superficial wound infection was observed in two $(12.5 \%)$ patients, and these patients were treated with superficial debridement and intravenous antibiotics.

The retrospective nature and small sample size were the major drawbacks of this study. Using different donor nerves (SAN, intercostal, phrenic) in limited number of patients impede interpretation of the results. In addition, different recepient tendons were used for gracilis tendon insertion. Prospective studies with longer follow-up may help us to better understand the long term effects of free gracilis flap on patient's functional status, perception of pain, and quality of life.

In conclusion, FGMF is a reliable option in the restoration of elbow flexion in patients with TBPP. Although there is an improvement in functional results, disability, and quality of life, there may be no change in patients' mental status and pain. Realistic targets should be established with the patients before surgery, and patients should be informed that the functionality may not be the same as before the injury.

\section{Declaration of conflicting interests}

The authors declared no conflicts of interest with respect to the authorship and/or publication of this article.

\section{Funding}

The authors received no financial support for the research and/or authorship of this article.

\section{REFERENCES}

1. Terzis JK, Kostopoulos VK. The surgical treatment of brachial plexus injuries in adults. Plast Reconstr Surg 2007;119:73e-92e.

2. Chuang DC. Neurotization procedures for brachial plexus injuries. Hand Clin 1995;11:633-45.

3. Narakas AO, Hentz VR. Neurotization in brachial plexus injuries. Indication and results. Clin Orthop Relat Res 1988;(237):43-56.

4. Songcharoen P, Mahaisavariya B, Chotigavanich C. Spinal accessory neurotization for restoration of elbow flexion in avulsion injuries of the brachial plexus. J Hand Surg Am 1996;21:387-90.

5. Doi K, Sakai K, Kuwata N, Ihara K, Kawai S. Reconstruction of finger and elbow function after complete avulsion of the brachial plexus. J Hand Surg Am 1991;16:796-803.

6. Doi K, Sakai K, Kuwata N, Ihara K, Kawai S. Double free-muscle transfer to restore prehension following complete brachial plexus avulsion. J Hand Surg Am 1995;20:408-14.

7. Carlsen BT, Bishop AT, Shin AY. Late reconstruction for brachial plexus injury. Neurosurg Clin N Am 2009;20:51-64.

8. Manktelow RT, Zuker RM. The principles of functioning 
muscle transplantation: Applications to the upper arm. Ann Plast Surg 1989;22:275-82.

9. Nicoson MC, Franco MJ, Tung TH. Donor nerve sources in free functional gracilis muscle transfer for elbow flexion in adult brachial plexus injury. Microsurgery 2017;37:377-82.

10. Yi Lee TM, Sechachalam S, Satkunanantham M. Systematic review on outcome of free functioning muscle transfers for elbow flexion in brachial plexus injuries. J Hand Surg Eur Vol 2019;44:620-7.

11. Atik OŞ. Is there something new and interesting in my article? Eklem Hastalik Cerrahisi 2019;30:69.

12. Coulet B, Boch C, Boretto J, Lazerges C, Chammas M. Free Gracilis muscle transfer to restore elbow flexion in brachial plexus injuries. Orthop Traumatol Surg Res 2011;97:785-92.

13. Akasaka $Y$, Hara T, Takahashi M. Restoration of elbow flexion and wrist extension in brachial plexus paralyses by means of free muscle transplantation innervated by intercostal nerve. Ann Chir Main Memb Super 1990;9:341-50.

14. Chung DC, Carver N, Wei FC. Results of functioning free muscle transplantation for elbow flexion. J Hand Surg Am 1996;21:1071-7.

15. Vekris MD, Beris AE, Lykissas MG, Korompilias AV, Vekris $\mathrm{AD}$, Soucacos PN. Restoration of elbow function in severe brachial plexus paralysis via muscle transfers. Injury 2008;39 Suppl 3:S15-22.

16. Terzis JK, Kostopoulos VK. Free muscle transfer in posttraumatic plexopathies part II: The elbow. Hand (N Y) 2010;5:160-70.

17. Maldonado AA, Romero-Brufau S, Kircher R N MF, Spinner RJ, Bishop AT, Shin AY. Free functioning gracilis muscle transfer for elbow flexion reconstruction after traumatic adult brachial pan-plexus injury: Where is the optimal distal tendon attachment for elbow flexion? Plast Reconstr Surg 2017;139:128-36.

18. Stevanovic M, Sharpe F. Functional free muscle transfer for upper extremity reconstruction. Plast Reconstr Surg 2014;134:257e-274e.

19. Kimura LK, do Nascimento AT, Capócio R, Mattar R Jr, Rezende MR, Wei TH, et al. Microsurgical transfer of the gracilis muscle for elbow flexion in brachial plexus injury in adults: Retrospective study of eight cases. Rev Bras Ortop 2015;46:534-9.

20. Doi K. Management of total paralysis of the brachial plexus by the double free-muscle transfer technique. J Hand Surg Eur Vol 2008;33:240-51.

21. Kay S, Pinder R, Wiper J, Hart A, Jones F, Yates A. Microvascular free functioning gracilis transfer with nerve transfer to establish elbow flexion. J Plast Reconstr Aesthet Surg 2010;63:1142-9.
22. Doi K, Hattori Y, Yamazaki H, Wahegaonkar AL, Addosooki A, Watanabe M. Importance of early passive mobilization following double free gracilis muscle transfer. Plast Reconstr Surg 2008;121:2037-45.

23. Barrie KA, Steinmann SP, Shin AY, Spinner RJ, Bishop AT. Gracilis free muscle transfer for restoration of function after complete brachial plexus avulsion. Neurosurg Focus 2004;16:E8.

24. Estrella EP, Montales TD. Functioning free muscle transfer for the restoration of elbow flexion in brachial plexus injury patients. Injury 2016;47:2525-33.

25. Dodakundi C, Doi K, Hattori Y, Sakamoto S, Fujihara Y, Takagi $\mathrm{T}$, et al. Outcome of surgical reconstruction after traumatic total brachial plexus palsy. J Bone Joint Surg [Am] 2013;95:1505-12.

26. Yang Y, Yang JT, Fu G, Li XM, Qin BG, Hou Y, et al. Functioning free gracilis transfer to reconstruct elbow flexion and quality of life in global brachial plexus injured patients. Sci Rep 2016;6:22479.

27. Mahmood B, Marshall D, Wolfe SW, Lee SK, Fufa DT. Anatomic evaluation of the obturator branch to the gracilis muscle to optimize its use as a free functional muscle transfer for elbow flexion reanimation. The Journal of Hand Surgery 2018;43:S50-S51.

28. Schreiber JJ, Byun DJ, Khair MM, Rosenblatt L, Lee SK, Wolfe SW. Optimal axon counts for brachial plexus nerve transfers to restore elbow flexion. Plast Reconstr Surg 2015;135:135e-141e.

29. Satbhai NG, Doi K, Hattori Y, Sakamoto S. Functional outcome and quality of life after traumatic total brachial plexus injury treated by nerve transfer or single/double free muscle transfers: A comparative study. Bone Joint J 2016;98B:209-17.

30. Bertelli JA. Free reverse Gracilis muscle combined with Steindler flexorplasty for elbow flexion reconstruction after failed primary repair of extended upper-type paralysis of the brachial plexus. J Hand Surg Am 2019;44:112-20.

31. Potter SM, Ferris SI. Reliability of functioning free muscle transfer and vascularized ulnar nerve grafting for elbow flexion in complete brachial plexus palsy. J Hand Surg Eur Vol 2017;42:693-9.

32. Hosseinian MA, Tofigh AM. Cross pectoral nerve transfer following free gracilis muscle transplantation for chronic brachial plexus palsy: A case series. Int J Surg 2008;6:125-8.

33. Adams JE, Kircher MF, Spinner RJ, Torchia ME, Bishop AT, Shin AY. Complications and outcomes of functional free gracilis transfer in brachial plexus palsy. Acta Orthop Belg 2009;75:8-13. 